\title{
Takotsubo Cardiomyopathy with a Dramatically Rapid Resolution
}

\author{
Muhammad Alichaudhry, Chadi Dib, Ralph Lazzara
}

Department of Cardiovascular Medicine, University of Oklahoma Health Sciences Center, Oklahoma City, USA.

Email: muhammad-chaudhry@ouhsc.edu

Received March 5 ${ }^{\text {th }}, 2011$; revised April 6 ${ }^{\text {th }}, 2011$; accepted April 19 ${ }^{\text {th }}, 2011$.

\begin{abstract}
Takotsubo cardiomyopathy is a relatively rare disease entity with a rapid reversible ventricular dysfunction, which usually occurs in post-menopausal women, and is frequently associated with a stressful event. Diagnosing Takotsubo cardiomyopathy can sometimes be challenging. The absence of coronary artery obstructive disease, and the dramatic presentation of the patient mimicking acute coronary syndrome, make this disease unique. We discuss here a case of Takotsubo cardiomyopathy with extremely rapid resolution in just two days, which we believe is being reported for the first time and touch briefly on the salient features, pathogenesis, diagnosis, treatment, prognosis, and clinical significance of this unique entity.
\end{abstract}

Keywords: Takotsubo Cardiomyopathy, Rapid Resolution

\section{Introduction}

Takotsubo cardiomyopathy (TTC) is a unique disease entity, with a characteristic reversible ventricular dysfunction, making its timely diagnosis quite often a daunting proposition. The ventricular dysfunction associated with this usual entity is often but not always characterized by apical akinesis, and basal hyperkinesis, in a distribution which does not correlate, with a specific, unique, epicardial coronary artery blood supply [1]. Here we discuss a case of Takotsubo cardiomyopathy with extremely rapid resolution, in just two days, which we believe, is being reported for the first time. We review briefly the essential features of this disease including epidemiology, pathogenesis, clinical presentation, treatment, and prognosis.

\section{Case Report}

A 62 year old lady, with past medical history of hypertension, and chronic obstructive pulmonary disease was admitted with sudden shortness of breath, and chest pain. A few days before this episode, her youngest daughter had died in a traffic accident. Just prior to admission, she experienced sudden and severe shortness of breath at night, along with unrelenting chest pain located in central lower chest area, with no radiation. The chest pain resolved within half an hour, but the shortness of breath persisted, and rapidly worsened.
Upon presentation to the emergency room (ER), her physical exam revealed a blood pressure of 119/87, pulse rate of $84 / \mathrm{min}$, oxygen saturation of $86 \%$ on room air, and body temperature of $97.1^{\circ} \mathrm{F}$. There were no murmurs, rubs or gallops, no crackles on chest auscultation, no jugular venous distension, and no pedal edema. She had a mild elevation in her cardiac enzymes (Troponin $=0.05$, CK-MB = 1.7, CK = 97), and her Brain-Natriuretic Peptide (BNP) level was 1400. Her laboratory work-up was otherwise within normal limits. Her EKG showed nonspecific ST-T changes, and QT prolongation, with a corrected QT interval (QTc) of 505 ms, and her chest X-Ray showed hyperexpanded lung fields, with flattened diaphragms.

Given her low oxygen saturations, and her shortness of breath, she was initially started on BIPAP (Bi-level positive airway pressure) and was given furosemide, aspirin, heparin infusion, and statin, with the most likely diagnosis being: heart failure secondary to Non-ST Elevation Myocardial Infarction (NSTEMI).

A Transthoracic Echocardiogram (TTE), was performed the next day, and showed marked left ventricular systolic dysfunction (ejection fraction of 15\% - 20\%), severe apical hypokinesis, basal hyperkinesis, moderately reduced right ventricular function, and mild mitral regurgitation (Figure 1). Left heart catheterization revealed normal coronary arteries, with no obstructive disease. 


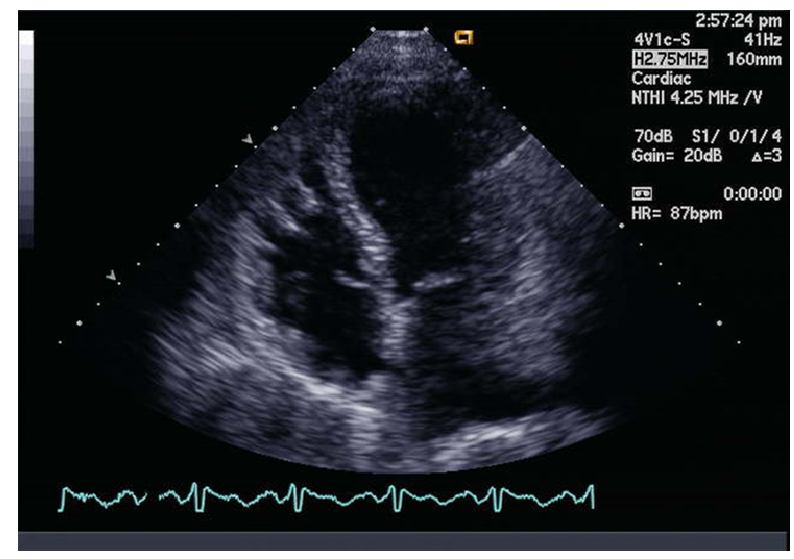

Figure 1. Characteristic apical ballooningin systole with markedly reduced left ventricular ejection fraction.

A repeat TTE was obtained, after two days, following a dramatic resolution of patient's symptoms. The latter revealed a normal systolic function with an ejection fraction of $55 \%-65 \%$ with absence of apical hypokinesis (Figure 2). The moderately reduced right ventricular function, and the mild mitral regurgitation, seen previously, had completely resolved, at the time, the second echocardiogram was done.

Interestingly, the nonspecific ST-T changes, and prolonged QTc on admission resolved as well, over a time period of two days.

\section{Discussion}

Stress-induced cardiomyopathy, also called apical ballooning syndrome, is generally characterized by transient systolic dysfunction of the apical, and/or mid segments of the left ventricle (LV), that mimics myocardial infarction, in the absence of significant coronary artery disease [1].

Typically, the contractile function of the mid, and apical segments, of the LV is depressed with compensatory

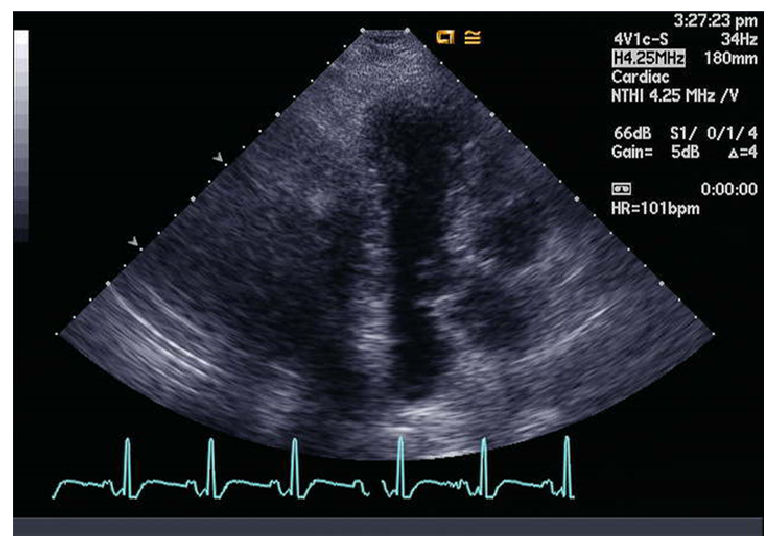

Figure 2. Resolution of the ballooning on the second day with normal left ventricular ejection fraction. hyperkinesis of the basal walls, producing "ballooning of the apex, during systole. A different type of transient cardiomyopathy, with mid-ventricular akinesis, has also been described (mid-ventricular ballooning syndrome). In a description, of six cases of mid-ventricular ballooning, there was mild ventricular dysfunction (ejection fraction $40 \%$ ), and shorter recovery times (1 - 7 days) [2]. However, in our case there was typical apical ballooning, with no mid-ventricular involvement, and a significant reduction in left ventricular ejection fraction.

Stress-induced cardiomyopathy, is much more common in women than men. In a review of ten prospective series, women accounted for $80 \%-100 \%$ of cases, with a mean age of onset, of $61-76$ years [3]. In a prospective registry of 3,265 patients,frequency of transient cardiomyopathy was $1.2 \%$ in troponin positive acute coronary syndrome (ACS) patients [4]. The onset of stress-induced cardiomyopathy, is frequently, but not always triggered by an acute medical illness, or by intense emotional or physical stress [5]. Postulated mechanisms include: catecholamine excess, coronary artery spasm, and microvascular dysfunction among others, which leads to myocardial stunning. Direct catecholamine toxicity has been implicated as well [6].

The clinical presentation, of stress-induced cardiomyopathy, is similar to that of an acute myocardial in- farction (MI) [7]. The most common presenting symptom is acute sub sternal chest pain, but some patients present with dyspnea, syncope, shock, or electrocardiographic (EKG) abnormalities [8].

The EKG often reveals ST elevation (mostly precordial) during the acute phase, followed by $\mathrm{T}$ wave inversion, QT prolongation, and sometimes deep Q waves during the subacute phase $[9,10]$. In a comparison study of takotsubo cardiomyopathy and acute myocardial infarction (MI) $34 \%$ to $56 \%$ of takotsubo patients showed ST elevation in anterior leads $[9,10]$, with absence of reciprocal changes, prolongation of QTc interval, and deep $\mathrm{T}$ wave inversion $[9,10]$. Cardiac biomarkers are usually mildly elevated [10]. Another unique aspect which has come to light is occasional involvement of the right ventricle (RV). In a series of 34 patients, with stress induced cardiomyopathy, it was found that $26 \%$ had RV motion abnormalities [11]. In our case, non specific ST-T wave changes, QTc prolongation, and moderate right ventricular dysfunction, were present on admission, and resolved over two days, along with resolution of left ven -tricular dysfunction.

Despite the severity of the acute illness, stress-induced cardiomyopathy is a transient disorder, managed with supportive therapy. Conservative medical treatment with beta-blockers when possible has been thought to be associated with lower mortality during the acute phase [12]. 
ACE inhibitors, and diuretics, are used as required with volume overload and aspirin in setting of coexisting coronary atherosclerosis. Resolution of the physical or emotional stress, usually results in rapid resolution of symptoms [13], and EKG changes.

In-hospital mortality rates have been reported by Gianni et al., as 1.1\%, along with a recurrence rate of 3.5\% [14]. In a prospective study of 136 patients with stress cardiomyopathy the mortality rate was $2 \%$ [15]. However, in another retrospective study of 100 patients, with a mean follow up of $4.4 \pm 4.6$ years the recurrence rate was $11.4 \%$, over 4 years after initial presentation [16].

The recovery times, for typical cases with apical ballooning, are highly variable. Patients who survive the acute episode, typically recover normal ventricular function within 1 - 4 weeks, as shown in a study of 30 patients, with takotsubo cardiomyopathy [17]. In this case, the ground for unexpectedly quick resolution, within two days, is difficult to pinpoint with certainty.

In a study of 22 patients, over a 32 month period the mean LVEF increased from $29 \%$ at initial presentation to $63 \%$ at a mean of 6 days [17]. In another study of 19 patients LVEF increased from a median of $20 \%$ at presentation to $60 \%$ in 2 - 4 weeks [18]. It should be noted though, that daily echocardiogram has not been performed, prospectively on patients admitted with takotsubo cardiomyopathy, and therefore the exact period of resolution, has not been well elucidated so far.

\section{Conclusions}

This case is to our knowledge, the first case that highlights the dramatic resolution of takotsubo cardiomyopathy, which occurred within two days of presentation. We believe this to be the most rapid EF normalization, ever published to date, for a case of takotsubo cardiomyopathy with typical apical ballooning pattern [19]. Extensive related search of literature to establish this was done, using 'takotsubo' and 'quick resolution' as the key words. We emphasize the fact that keen insight of physicians is extremely important, as to not overlook the highly diverse clinical scenarios, and unpredictable patterns of this unique disease. This also warrants the need, for future studies to be done, looking at prognostic variables that could be associated with the duration of left ventricular dysfunction.

\section{REFERENCES}

[1] A. Prasad, A. Lerman and C. SRihal, “Apical Ballooning Syndrome (Tako-Tsubo or Stress Cardiomyopathy): A Mimic of Acute Myocardial Infarction," American Heart Journal, Vol. 155, No. 3, 2008, pp. 408-417. doi:10.1016/j.ahj.2007.11.008

[2] J. M. Aubert, P. V. Ennezat, O. Tricot, J. Darchis, J. J.
Bauchart and J. L. Auffray, et al., "Mid-ventricular Ballooning Heart Syndrome,” Echocardiography, Vol. 24, No. 4, 2007, pp. 329-334.

[3] Y. J. Akashi, D. S. Goldstein, G. Barbaro and T. Ueyama, et al., "Takotsubo Cardiomyopathy: A New Form of Acute, Reversible Heart Failure,” Circulation, Vol. 118, No. 25, 2008, pp. 2754-2762. doi:10.1161/CIRCULATIONAHA.108.767012

[4] K. Tsuchihashi, K. Ueshima, T. Uchida, N. Oh-mura, K. Kimura and M. Owa, et al., "Transient Left Ventricular Apical Ballooning without Coronary Artery Stenosis: a Novel Heart Syndrome Mimicking Acute Myocardial Infarction,” Angina Pectoris-Myocardial Infarction Investigations in Japan, Journal of the American College of Cardiology, Vol. 38, No. 1, 2001, pp. 11-18. doi:10.1016/S0735-1097(01)01316-X

[5] V. Kurowski, A. Kaiser, K. von Hof, D. P. Killermann, B. Mayer and F. Hartmann, et al., "Apical and Midventricular Transient Left Ventricular Dysfunction Syndrome (TakoTsubo Cardiomyopathy): Frequency, Mechanisms and Prognosis,” Chest, Vol. 132, No. 33, 2007, pp. 809-816. doi:10.1378/chest.07-0608

[6] H. M. Nef, H. Mollmann, S. Kostin, C. Troidl, S. Voss , and M. Weber, et al., "Tako-Tsubo Cardiomyopathy: Intraindividual Structural Analysis in the Acute Phase and after Functional Recovery,” European Heart Journal, Vol. 28, No. 20, 2007, pp. 2456-2464. doi:10.1093/eurheartj/ehl570

[7] K. A. Bybee, T. Kara, A. Prasad,A. Lerman, G. W. Barsness and R. S. Wright, et al., "Systematic Review: Transient Left Ventricular Apical Ballooning: A Syndrome that Mimics ST-Segment Elevation Myocardial Infarction," Annals of Internal Medicine, Vol. 141, No. 11, 2004, pp. 858-865.

[8] W. J. Desmet, B. F. Adriaenssens and J. A. Dens, “Apical Ballooning of the Left Ventricle: First Series in White Patients,” Heart, Vol. 89, No. 9, 2003, pp. 1027-1031. doi:10.1136/heart.89.9.1027

[9] R. Ogura, Y. Hiasa, T. Takahashi, K. Yamaguchi, K. Fujiwara and Y. Ohara, et al., "Specific Findings of the Standard 12-lead ECG in Patients with 'Takotsubo' Cardiomyopathy: Comparison with the Findings of Acute Anterior Myocardial Infarction,” Circulation Journal, Vol 67, No. 8, 2003, pp. 687-690. doi:10.1253/circj.67.687

[10] S. W. Sharkey, J. R. Lesser, M. Meno, M. Parpart, M. S. Maron and B. J. Maron, et al., "Spectrum and Significance of Electrocardiographic Patterns, Troponin Levels, and Thrombolysis in Myocardial Infarction Frame Count in Patients with Stress (Tako-Tsubo) Cardiomyopathy and Comparison to Those in Patients with St-Elevation Anterior Wall Myocardial Infarction,” American Journal of Cardiology, Vol. 101, No. 12, 2008, pp. 1723-1728. doi:10.1016/j.amjcard.2008.02.062

[11] C. Dib, S. Asirvatham, A. Elesber, C. Rihal, P. Friedman, and A. Prasad, et al., "Clinical Correlates and Prognostic Significance of Electrocardiographic Abnormalities in Apical Ballooning Syndrome (Takotsubo/Stress-Induced 
Cardiomyopathy),” American Heart Journal, Vol. 157, No. 5, 2009, pp. 933-938. doi:10.1016/j.ahj.2008.12.023

[12] C. Dib, A. Prasad, P. A. Friedman, E. Ahmad, C. S. Rihal and S. C. Hammill, et al., "Malignant Arrhythmia in Apical Ballooning Syndrome: Risk Factors and Outcomes," Indian Pacing and Electrophysiology Journal, Vol. 8, No. 3, 2008, pp. 182-192.

[13] K. A. Bybee and A. Prasad, "Stress-Related Cardiomyopathy Syndromes,” Circulation, Vol. 118, No. 4, 2008, pp. 397-409. doi:10.1161/CIRCULATIONAHA.106.677625

[14] M. Gianni, F. Dentali, A. M. Grandi, G. Sumner, R. Hiralal and E. Lonn, et al., "Apical Ballooning Syndrome or Takotsubo Cardiomyopathy: A Systematic Review," European Heart Journal, Vol. 27, No. 13, 2006, pp. 1523-1529. doi:10.1093/eurheartj/ehl032

[15] S. W. Sharkey, D. C. Windenburg, J. R. Lesser, M. S. Maron, R. G. Hauser and J. N. Lesser, et al., "Natural History and Expansive Clinical Profile of Stress (TakoTsubo) Cardiomyopathy," Journal of the American College of Cardiology, Vol. 55, No. 4, 2010, pp. 333-341. doi:10.1016/j.jacc.2009.08.057

[16] A. A. Elesber, A. Prasad, R. J. Lennon, R. S. Wright, A. Lerman and C. S. Rihal, et al., "Four-Year Recurrence Rate and Prognosis of the Apical Ballooning Syndrome," Journal of the American College of Cardiology, Vol. 50, No. 5, 2007, pp. 448-452. doi:10.1016/j.jacc.2007.03.050

[17] S. W. Sharkey, J. R. Lesser, A. G. Zenovich, M. S. Maron, J. Lindberg and T. F. Longe, et al., "Acute and Reversible Cardiomyopathy Provoked by Stress in Women from the United States," Circulation, Vol. 111, No. 4, 2005, pp. 1472-1479. doi:10.1161/01.CIR.0000153801.51470.EB

[18] I. S. Wittstein, D. R. Thiemann, J. A. C. Lima, K. L. Baughman, S. P. Schulman and G. Gerstenblith, et al., "Neurohumoral Features of Myocardial Stunning due to Sudden Emotional Stress," The New England Journal of Medicine, Vol. 352, No. 6, 2005, pp. 539-548. doi:10.1056/NEJMoa043046

[19] S. Kumar, N. Mostow and A. Grimmr, "Quick Resolution of Takotsubo Cardiomyopathy: A Brief Review,” Echocardiography, Vol. 25, No. 10, 2008, pp. 1117-1120. doi:10.1111/j.1540-8175.2008.00775.x 